\title{
LOS VIENTOS DE LEVANTE EN EL ESTRECHO DE GIBRALTAR EN EL VERANO DE 2016
}

\author{
J. M. Sánchez-Laulhé \\ Centro Meteorológico de Málaga. AEMET, jsanchezlaulheo@aemet.es
}

\section{RESUMEN}

El verano de 2016 se caracterizó por el gran número de días con temporal de viento de levante en el estrecho de Gibraltar, especialmente los meses de julio y agosto. A pesar de que en ese verano la medida del viento falló en horas diurnas en la estación de Tarifa, se estima, a partir los datos nocturnos, un recorrido del viento en agosto de más de $20000 \mathrm{~km}$ en dicha estación, lo que supone el agosto con mayor recorrido del siglo XXI. Para comparar los datos con los del siglo XX se hace otra corrección por el cambio de emplazamiento que sufrió la estación en el año 2000, que llevaría a un recorrido de más de 25000 km si la estación hubiera permanecido en su ubicación antigua, lo que supondría uno de los agostos con más viento de levante de la serie de Tarifa.

Hay que resaltar en primer lugar que mientras en el siglo XX el periodo de retorno de un "agosto de levante" en el Estrecho (recorrido del viento mayor de $25000 \mathrm{~km}$ ) era de unos 5 años, a partir de 1998 no hubo ningún "agosto de levante" hasta el 2016, y en segundo lugar que estos hayan ocurrido inmediatamente tras desaparecer los fenomenos El Niño de 1997-1998 y de 2015-2016, que han sido los más fuertes de la historia.

Se ha calculado, para el periodo 1995-2016, la correlación entre la presión a nivel del mar en agosto y el índice Niño3.4 de meses anteriores, existiendo una zona de correlación superior al 50 \% en la zona que se extiende desde las islas británicas hasta las islas Baleares y el sudeste de la Península (ver figura 1). Esta correlación no se mantiene al considerar periodos anteriores a 1995.

\section{Los vientos en la estación de AEMET de Tarifa}

El verano de 2016 se ha caracterizado por el gran número de días con temporal de levante en el estrecho de Gibraltar, especialmente los meses de julio y agosto. Sin duda los meses de verano con más levante del presente siglo (Sánchez-Laulhé, 2017). En la figura 1 se muestra el recorrido del viento en los meses de agosto (distancia que recorrería una partícula a la velocidad del viento medido en la estación durante el mes) en la estación meteorológica de Tarifa, desde el año 1946 al 2016. El recorrido del viento en esta estación en verano está estrechamente relacionado a la frecuencia de los temporales de levante. La estación de Tarifa sufrió un cambio de emplazamiento en el año 2000 en el que el viento es menor, como salta a la vista en la gráfica, pues está más alejado del mar, el anemómetro está más bajo y además tiene un pequeño cerro al este. Además, este verano la medida del viento ha sido errónea en horas diurnas, por lo que el recorrido del viento se ha estimado a partir de las medidas nocturnas, con un resultado de más de $20000 \mathrm{~km}$, lo que ya supone el agosto con mayor recorrido del siglo XXI. Si, para poder comparar los datos de este año con los del siglo XX le hacemos una corrección aproxi- 
mada que contrarreste el cambio de emplazamiento, el recorrido del viento superaría los $25000 \mathrm{~km}$ (en verde en la figura 1), valor que podríamos considerar un umbral para un "agosto de levante". Es llamativo que mientras en el siglo XX el periodo de retorno de un agosto de levante fue de unos 5 años, a partir de 1998 no ha habido ningun agosto de levante hasta el 2016 (18 años después).

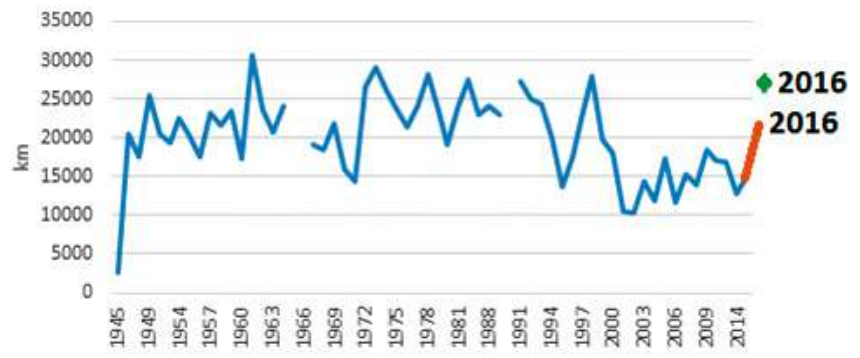

Figura 1. Recorrido del viento en los meses de agosto medidos en la estación meteorológica de Tarifa de AEMET.

\section{El campo medio de presión en agosto}

La figura 2 muestra un mapa del campo de presión a nivel del mar media de agosto de 2016 centrado en el mar Mediterráneo.

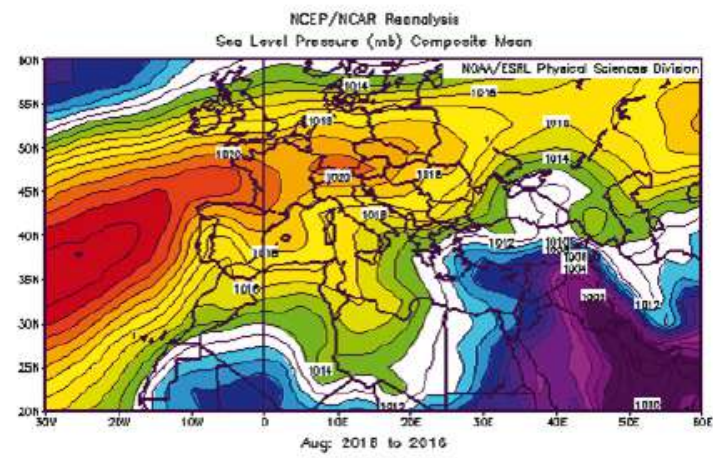

Figura 2. Campo de presión a nivel del mar media de agosto de 2016. Mapa obtenido de los reanálisis del NCEP/NCAR.

La distribución de la presión a grandes rasgos se corresponde a la que se espera para los meses de verano, estación en que los anticiclones subtropicales adquieren su máxima intensidad media y están localizados en las cuencas oceánicas, presentando en el Este un notable gradiente de presión perpendicular a la costa al que está asociado un viento relativamente fuerte con gran componente meridional dirigido hacia el ecuador, (alisio canario). Estas características también aparecen en el este del Mediterráneo vientos de componente norte, etesios sobre el mar Egeo, que penetran sobre zonas de desierto del norte de África, entre una cuña anticiclónica con eje en Túnez y las bajas presiones sobre el este del Mediterráneo, que prolongan la vaguada pérsica, que emana de la enorme baja de calor del sur de Asia. 
NCEP/NCAR Rennalysia

Sea Level Pressure (mb) Composite Anomaly 1981-2010 climo
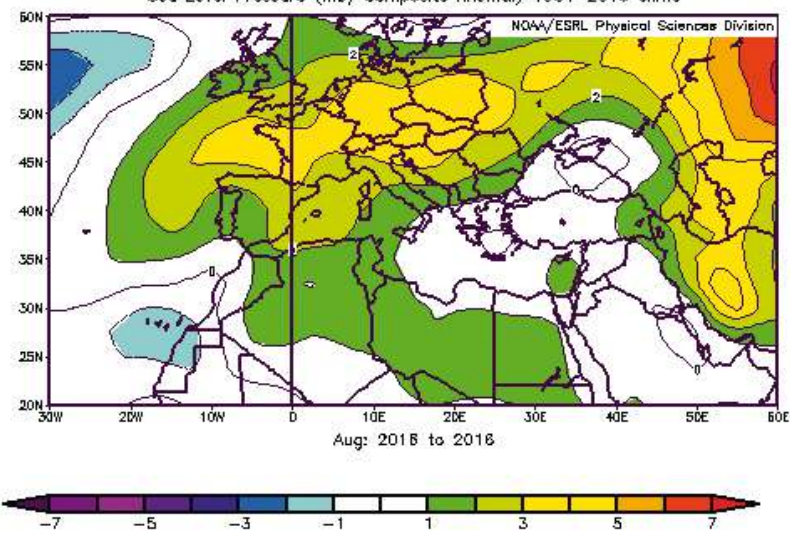

Figura 3. Anomalías medias de la presión a nivel del mar de agosto 2016 respecto a la media de agosto del periodo 1981-2010. Mapa obtenido de los reanálisis del NCEP/NCAR.

En el mapa de presión de agosto también se observa que el anticiclón de las Azores se extiende hacia Centroeuropa y hacia el Mediterráneo occidental, haciendo subsidiaria la cuña anticiclónica de Túnez. Estas extensiones son los elemen-

tos principales del mapa de anomalía de presión que se muestra en la figura 3, en el que además se puede apreciar que toda Europa occidental, y buena parte de África y Asia, tienen una anomalía positiva de presión. Dejando a un lado el Atlántico al oeste de las islas británicas, solamente sobre las islas Canarias existe anomalía negativa mayor que $1 \mathrm{mb}$, que se extiende más débilmente hacia el continente africano en dirección SE, y hacia el NE por las costas de Marruecos. Sobre los mares Negro y Azov y la península de Crimea existe otra pequeña anomalía negativa menor que no alcanza $-1 \mathrm{mb}$. Quizá parte de la preponderancia de las anomalías se deba a la anomalía positiva casi global de temperatura. En la figura 4 muestra que la correlación entre la presión a nivel del mar en agosto y la temperatura global es positiva sobre las zonas continentales, y negativa sobre el centro

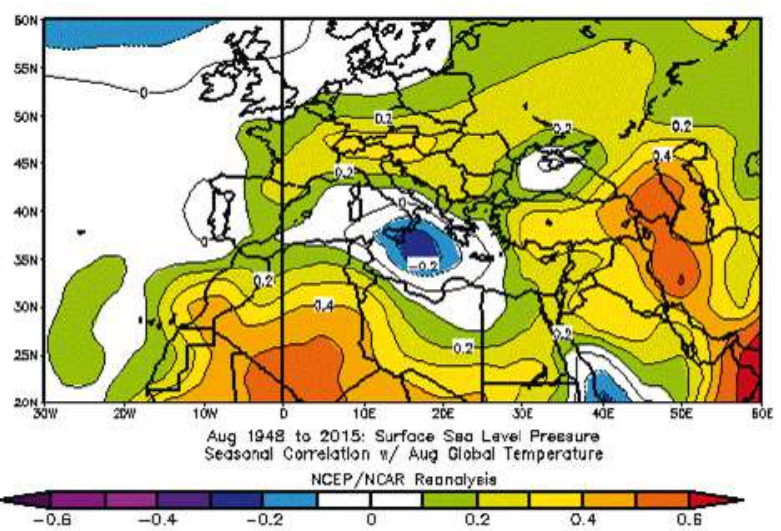
del Mediterráneo. Esta correlación es relativamente alta sobre el interior de África, Persia y el Cáucaso.

Figura 4. Correlación media en agosto de la presión a nivel del mar y la temperatura global para el periodo 1948-2015, obtenida de los reanálisis del NCEP/NCAR.

Del mapa de anomalía de presión se infiere: la extensión hacia buena parte del Mediterráneo occidental del viento de componente norte, y por tanto también del área con velocidad vertical descendente (Rodwell y Hoskins, 2002); la existencia de un fuerte gradiente de presión meridional con isobaras y vientos paralelos a la costa de Argelia; en consecuencia en 
el mapa de presión (figura 2) las isobaras se disponen transversalmente a los mares de Palos y Alborán acelerando el flujo de levante hacia el Estrecho de Gibraltar.

\section{Vientos de levante y El Niño}

Los temporales de levante en el Estrecho pueden ser de escala mesoescalar o de escala sinóptica (Dorman et al, 1995). Los primeros están muy localizados y afectan especialmente en Estrecho, no llegando a alcanzar a la capital de Cádiz ni al mar de Alborán, y se trata simplemente de salida de aire frío por el estrecho de Gibraltar, y son los temporales más frecuentes en verano . Los sinópticos sin embargo afectan a buena parte del sur de la Península. Esta diferencia de escalas es importante cuando se trata de buscar relaciones con índices climáticos a través de los reanálisis en los que solo se reflejan los de escala sinóptica. Así la figura 5, en la que está representado el viento zonal medio en agosto en la zona del Estrecho al nivel de $925 \mathrm{hPa}$ para el periodo 1948-2016 obtenido de los reanálisis del NCEP (valores negativos corresponden a vientos de levante), muestra solo cuatro de los años con viento de levante mayor de $1 \mathrm{~m} / \mathrm{s}$, siendo el máximo el de

viento zonal medio en agosto en el entorno del Estrecho (reanálisis NCEP)

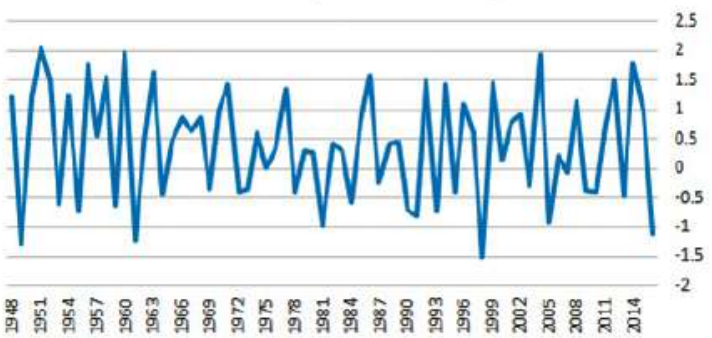

1998, y el de 2016 el único del siglo XXI.

Figura 5. Viento zonal medio en agosto en la zona del estrecho de Gibraltar en $925 \mathrm{hPa}$ para el periodo 1948-2016 obtenido de los reanálisis del NCEP (valores negativos corresponden a vientos de levante).

Los "Niños" de 1997-1998

y de 2015-2016 (figura 6) han sido los más fuertes de la historia, lo cual nos lleva a considerar si este fenómeno tiene alguna relación con los agostos de levante.

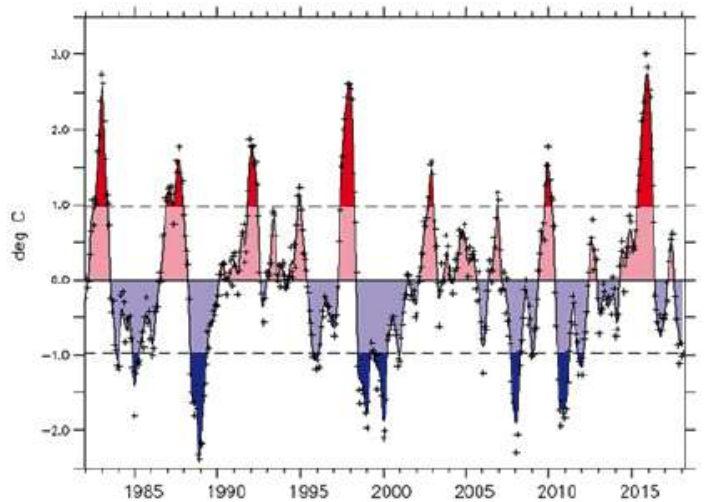

Figura 6. Índice Niño3.4 desde 1982 hasta el presente. Fuente NOAA

Teniendo en cuenta que durante los agostos de 1998 y 2016 ya había desaparecido El Niño, lo lógico es explorar correlaciones entre la presión a nivel del mar en agosto y los índices El Niño de meses anteriores. Considerando el índice Niño3.4 de 6 meses antes y para el periodo 1995-2016, se 
obtiene el mapa de la figura 7 en la que se aprecia una zona de correlación superior al 50\% en la zona que se extiende desde las islas británicas hasta las islas Baleares y el sudeste de la Península. Hay que advertir que las correlaciones de estos mismos parámetros para periodos anteriores a 1995 son mucho más bajas y no congruentes con las de la figura 7, por lo que se podría pensar que la correlación es alta solo para fenómenos El Niño muy intensos.

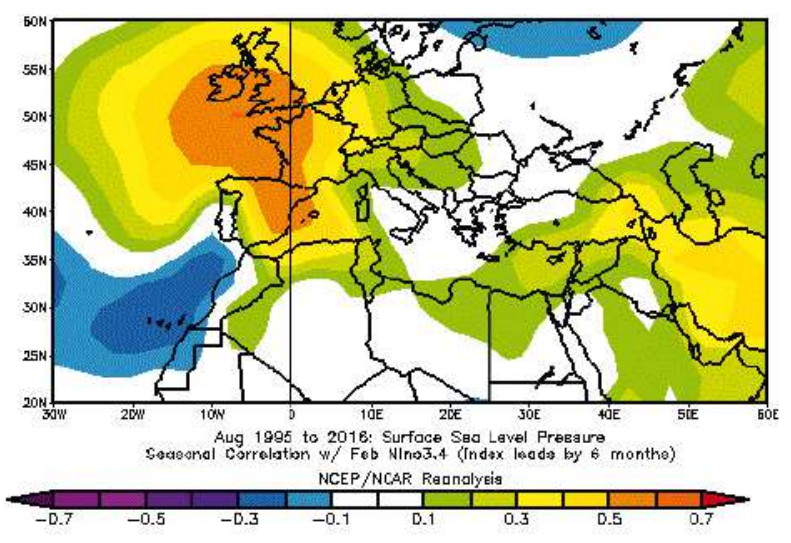

Figura 7. Correlación entre la presión a nivel del mar de agosto y el índice Niño 3.4 del mes de febrero anterior.

En la figura 8 se muestra el mapa de anomalías de la función de corriente media en $250 \mathrm{hPa}$ de agosto que muestra la onda de Rossby estacionaria con anomalía positiva (anticiclónica) sobre el Cantábrico y anomalía negativa (ciclónica) con centro en Túnez, que habría dado lugar a la persistente situación de levante.

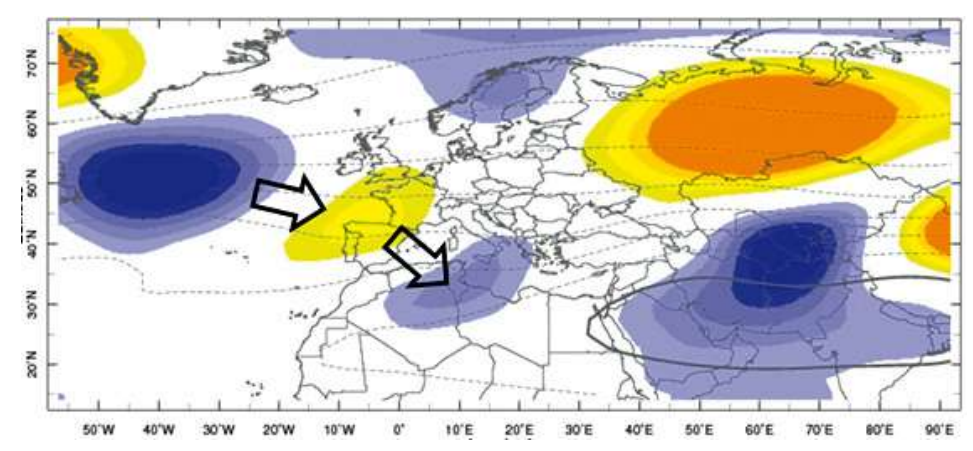

Figura 8. Mapa de función de corriente media en 250 hPa en agosto 2016 (a trazos) y de sus anomalías (en colores: azules las anomalías negativas; amarillas-anaranjadas las anomalías positivas). Las flechas marcan una onda de Rossby estacionaria.

\section{Referencias:}

- Dorman, C.E. , Beardsley R.C. , Limeburner R. 1995: Winds in the Strait of Gibraltar. Quarterly Journal of the Royal Meteorological Society. 121,1903-192.

- Sánchez-Laulhé, J.M. 2017: Mapa de presión a nivel del mar de agosto de 2016: verano de levantes. Tiempo y Clima. No 55, 34-36.

- Rodwell, M. J. y B. J. Hoskins, 2001: Subtropical anticyclones and summer monsoons. J. Climate, 14, 3192-3211. 